\title{
Environment and Brain Development: Challenges in the Global Context
}

\section{Citation}

Jordi Julvez, Tomas Paus, David Bellinger, Brenda Eskenazi, Henning Tiemeier, Neil Pearce, Beate Ritz, Tonya White, Paul Ramchandani, Juan Domingo Gispert, Sylvane Desrivières, Rachel Brouwer, Olivier Boucher, Silvia Alemany, Mónica López-Vicente, Elisabeth Suades-González, Joan Forns, Philippe Grandjean, Jordi Sunyer. Environment and Brain Development: Challenges in the Global Context. Neuroepidemiology. 2016;46(2):79-82. doi: 10.1159/000442256.

\section{Published Version}

doi:10.1159/000442256

\section{Permanent link}

http://nrs.harvard.edu/urn-3:HUL.InstRepos:37221740

\section{Terms of Use}

This article was downloaded from Harvard University's DASH repository, and is made available under the terms and conditions applicable to Open Access Policy Articles, as set forth at http:// nrs.harvard.edu/urn-3:HUL.InstRepos:dash.current.terms-of-use\#OAP

\section{Share Your Story}

The Harvard community has made this article openly available.

Please share how this access benefits you. Submit a story.

Accessibility 


\section{Environment and Brain Development: Challenges in the Global Context}

Jordi Julvez ${ }^{1}$, Tomas Paus ${ }^{2}$, David Bellinger ${ }^{3}$, Brenda Eskenazi ${ }^{4}$, Henning Tiemeier ${ }^{5}$, Neil Pearce ${ }^{6}$, Beate Ritz ${ }^{7}$, Tonya White ${ }^{8}$, Paul Ramchandani ${ }^{9}$, Juan Domingo Gispert $^{10}$, Sylvane Desrivières ${ }^{11}$, Rachel Brouwer ${ }^{12}$, Olivier Boucher ${ }^{13}$, Silvia Alemany ${ }^{1}$, Mónica López-Vicente ${ }^{1}$, Elisabeth Suades-González', Joan Forns ${ }^{1}$, Philippe Grandjean ${ }^{14}$ and Jordi Sunyer ${ }^{1}$

1) Centre for Research in Environmental Epidemiology, Barcelona, Catalonia. Spain

2) The Rotman Research Institute, Departments of Psychology and Psychiatry, University of Toronto, Toronto, Ontario, Canada; Child Mind Institute, New York, United States of America

3) Department of Psychiatry, Harvard Medical School and Boston Children's Hospital, Boston, MA, United States of America.

4) Center for Environmental Research and Children's Health (CERCH), School of Public Health, University of California, Berkeley, CA, United States of America.

5) Department of Psychiatry, Erasmus Medical Centre, Rotterdam, the Netherlands.

6) London School of Hygiene and Tropical Medicine, London, United Kingdom.

7) Department of Epidemiology, University of California at Los Angeles School of Public Health, Los Angeles, CA, United States of America.

8) Department of Child and Adolescent Psychiatry/Psychology, Erasmus MCSophia Children's Hospital, Rotterdam, the Netherlands. 
9) Unit of Child and Adolescent Psychiatry, Imperial College London, London, United Kingdom.

10) Fundació Pasqual Maragall, Plataforma de Neuroimatge, Barcelona, Catalonia, Spain.

11) SGDP Centre, Institute of Psychiatry, Psychology and Neuroscience, King's College London, London, United Kingdom.

12) Department of Psychiatry, Brain Center Rudolf Magnus, University Medical Center Utrecht, Utrecht, the Netherlands.

13) Centre de Recherche en Neuropsychologie et Cognition, Département de Psychologie, Université de Montréal, Montréal, Que., Canada.

14) Department of Environmental Health, Harvard School of Public Health, Boston, MA, United States of America. 
The human brain develops over an extended period; its maturation continues through adolescence and young adulthood (1, 2, 3). Studying trajectories of brain development in representative samples of the general population is important in order to understand exposures and stressors in the child's and adult's physical and social environment that shape human brain development (2, 3, 4). Early environments may be particularly important in their impact on mental health, learning and behaviour in human societies $(2,3)$. In this context, it is important - for both theoretical and practical reasons - to measure trajectories of brain development in large population-based epidemiological studies $(2,5)$.

Researchers with expertise in environmental epidemiology, neuropsychology, psychiatry and developmental cognitive neuroscience contributed to a two-day scientific debate convened in Barcelona during October 2014. The debate focussed on neuroimaging and neuropsychological approaches for the assessment of brain and cognition in typically developing children and adolescents, and the challenges of assessing environmental exposure for studies carried out in the general population. The ultimate goal was to generate a consensus about the importance of populationbased studies that integrate information across different levels: molecular (e.g., biochemical, genetic), systems (e.g., structural and functional neuroimaging and cognitive assessments) and populations (e.g., air pollution) (2). The debate covered three strategic areas: a) environmental pollution and population science; b) measures of brain development; and, c) future directions and conclusions. 


\section{Environmental Pollution and Population Science}

There are about 214 chemicals that have been documented in clinical and epidemiological studies as having neurotoxic properties, mainly in adults. Only 12 of these have been properly examined with regards to their effects on human brain development; this is because most of the other chemicals have not been explored specifically in pregnant women and children, and/or we have only limited data on exposures to these chemical at the population level. The evidence available on these 12 substances suggests that adverse impacts on brain development can happen at much lower exposures than those that affect the mature brain (3). Nonetheless, the present documentation (3) almost certainly underestimates the real number of chemicals affecting neurodevelopment. In consequence, there is a need to develop screening methods that are validated against epidemiologic data in their prediction of neurotoxicity. It has been hypothesized that many untested neurotoxic chemicals may be responsible for a "silent pandemic", in which early life exposures are causing multiple neurodevelopmental disorders, costing billions of dollars annually to our societies (3). For example, ambient air pollution is not yet listed in European Environmental Agency (EEA) as a neurotoxic hazard due to the complexity in measuring a mixture of exposure components. And yet, recent findings indicate negative relationships between cognitive performance and air pollution in school-age children (6). Similar problems in relation to exposure measurements exist for other environmental chemicals, such as persistent organic compounds (POPs) including organochlorinated and brominated compounds, or endocrine disruptors such as phthalates and phenols, which are mixtures of different highly correlated compounds (3). Multiple biological pathways and modes of action can help to explain the neurotoxicity of these and other environmental pollutants, from enhancing pro- 
inflammatory and oxidative stress mechanisms to endocrine system disruption (3). Parallel concerns about social environments and stresses reflect their importance for brain development; it is recognized that adverse exposures, such as child maltreatment, poverty of the learning environment, poor and inconsistent parenting, can all disrupt child's mental health on their own and in interaction with pollutant hazards $(3,7)$. Both the environment and neurodevelopment are complex, and studying the interaction of the chemical and non-chemical factors requires a multimodal approach that takes into account several dimensions in terms of measurements, including time (longitudinal approach with repeated exams) and space (neighborhoods, personal space), as well as co-exposures within and between physical and social environments. Taking such an integrated approach is important given that some co-exposures may have opposing effects, some positive other negative, on the outcomes; for example Omega-3 fatty acids vs. methylmercury (or PCBs) in seafood, or physical activity vs. exposure to ambient air pollution while exercising. Finally, even a weak effect on neurodevelopment is of large concern when the exposure is ubiquitous across populations. As pointed out by Geoffrey Rose "the majority of cases in the population occur not in the small numbers at very high risk but in the centre of the population distribution, where large numbers of people are exposed, albeit with only modest increases in risk." (8). Thus, reducing even slightly exposures of the general population to various risks present in their physical and social environments - especially during development - is likely to accrue large benefits for public health. 


\section{Measures of Brain Development}

Longitudinal measures of brain development - whether neuropsychological or neuroimaging - provide insights into typical trajectories against which one can evaluate the possible impact of adverse physical or social environments. Multimodal Magnetic Resonance Imaging (MRI) is non-invasive and provides detailed information about brain structure and function (2). For example, a recent MRI study has identified an association between prenatal exposure to air pollutants (polycyclic aromatic hydrocarbons) and the development of brain white-matter, cognition, and behaviour (9). Similarly, computerized neuropsychological tests performed repeatedly over time have shown association with air pollution in school-age children (6). Moreover, the recent inclusion of computerized tests has reduced inter-observer variability during assessment and such neuropsychological functions are recorded automatically preventing errors in data collection (6). It will be important in global health epidemiological studies to select an appropriate range of tests to assess complex cognitive functions (e.g., cognitive control or emotion regulation), as well as memory, perceptual and motor functions, with minimal cross-cultural biases. In this selection, we should go beyond WHO's recommended neurobehavioral core test battery (NCTB) which includes mostly basic cognitive functions (10). Furthermore, such "normative" outcome measures can be complemented by internationally standardized (behavioral) rating scales aimed at covering clinical outcome measures in order to improve our understanding of the relationship between environmental exposures and mental health. This approach relies on using a dimensional rather than a categorical approach when clinically diagnostic data are not available. The careful selection and combination of some of these measurements can make the understanding of neurodevelopment in populations feasible in a global context. 


\section{Future Directions and Conclusions}

There is a need to address one of the most important emerging and newly recognized scientific challenges in global public health: the study of environment and brain development. We need tools for state-of-the-art measurements for both (environmental) exposures and (brain) outcomes to provide knowledge necessary for future interventional/prevention trials in a global context. We agree that there is some need for general 'harmonization' of neuropsychological measurements and imaging across cultures and studies. Nonetheless, we should go beyond WHO's NCTB due to: 1) improved understanding of the vulnerability of the developing brain; 2) new insights into the sensitivity and validity of neuropsychological tests; and 3) improved and less expensive neuropsychologic, neurophysiologic and imaging methods. Nowadays, there is enough scientific and technological expertise available to adopt common outcome assessments when building successful global consortia. The research community needs to be sensitive to contextual factors that may influence the practicality and feasibility of different approaches. These include, for example, whether or not a medical center is close to a study population and how cultural and economic differences or parental education may influence participation. Furthermore, some of the brain mapping tools (e.g., MRI) are still expensive and require major logistic efforts, including standard data capture and complex data processing protocols. Other tools (e.g., electroencephalography [EEG], event-related potentials (ERPs)) are more cost effective and applicable in global context. In the future we can benefit more from the use of new and cheaper technologies, not only techniques and software that improve imaging and data processing, but lighter and portable EEG instruments, also due to the expanding use of smart phones and micro sensor 
technologies to capture, for example, a child's air pollution exposure, physical activity and cognitive functioning.

If we are to understand brain development and its determinants in their full diversity, we need to strive towards global standards for assessing and characterizing normal brain maturation. Achieving a consensus on optimal assessment approaches would enhance the collaboration across studies carried out in different linguistic, cultural and economic environments. Population science targeting child neurodevelopment and mental health is relevant for framing the global burden of non-communicable disease debate. In other words, the discussion initiative tries to reach a common goal of understanding forces underlying the brain development trajectories assessed in global and diverse context. The panel discussants concluded that it is crucial to include experts in neuropsychology, neuroimaging, developmental cognitive neuroscience, environmental epidemiology and exposure sciences in international consortia assessing the global health burden. 
KEY MESSAGES

- The study of neurodevelopment trajectories in a global context needs a multidisciplinary scientific approach in order to understand better human brain function and structure and its interaction with the environment.

- Mental health should not be understood as the absence of medical diagnoses but must be linked to optimal brain functioning.

- The careful selection of neuropsychological and neuroimaging measurements makes the understanding of neurodevelopment in populations feasible in a global context.

- Computer-based neuropsychological tests with low cross-cultural bias are important new tools in a global context.

- Common brain mapping approaches can be used to understand mechanistic pathways.

- Future research should take advantage of technology adding and improving measures of exposures and outcomes.

- International consortia conducting population based studies are a key tool. 


\section{References}

1) Bale TL. Epigenetic and transgenerational reprogramming of brain development. Nat Rev Neurosci. 2015 Jun;16(6):332-44.

2) Paus T. Population Neuroscience. Springer-Verlag Berlin Heidelberg 2013, ISBN: 978-3-642-36449-5 (Print), 978-3-642-36450-1 (Online).

3) Grandjean P, Landrigan PJ. Neurobehavioural effects of developmental toxicity. Lancet Neurol. 2014 Mar;13(3):330-8.

4) Chen JC, Wang X, Wellenius GA, Serre ML, Driscoll I, Casanova R, McArdle JJ, Manson JE, Chui HC, Espeland MA. Ambient air pollution and neurotoxicity on brain structure: Evidence from women's health initiative memory study. Ann Neurol. 2015 Sep;78(3):466-76.

5) Paus T. A primer for brain imaging: a tool for evidence-based studies of nutrition? Nutr Rev. 2010 Nov;68 Suppl 1:S29-37.

6) Sunyer J, Esnaola M, Alvarez-Pedrerol M, Forns J, Rivas I, López-Vicente M, Suades-González E, Foraster M, Garcia-Esteban R, Basagaña X, Viana M, Cirach M, Moreno T, Alastuey A, Sebastian-Galles N, Nieuwenhuijsen M, Querol X. Association between traffic-related air pollution in schools and cognitive development in primary school children: a prospective cohort study. PLoS Med. 2015 Mar 3;12(3):e1001792.

7) Hair NK et al (2015). Association of Child Poverty, Brain Development, and Academic Achievement. JAMA Pediatrics. Published online July 20, 2015. doi:10.1001/jamapediatrics.2015.1475.

8) Rose GA, Khaw KT, Marmot MG. Rose's Strategy of Preventive Medicine: The Complete Original Text. - 2008 - $171 \mathrm{p}$

9) Peterson BS, Rauh VA, Bansal R, Hao X, Toth Z, Nati G, Walsh K, Miller RL, Arias $F$, Semanek D, Perera F. Effects of prenatal exposure to air pollutants (polycyclic aromatic hydrocarbons) on the development of brain white matter, cognition, and behavior in later childhood. JAMA Psychiatry. 2015 Jun;72(6):531-40.

10) Anger WK. Reconsideration of the WHO NCTB strategy and test selection. Neurotoxicology. 2014 Dec;45:224-31. 